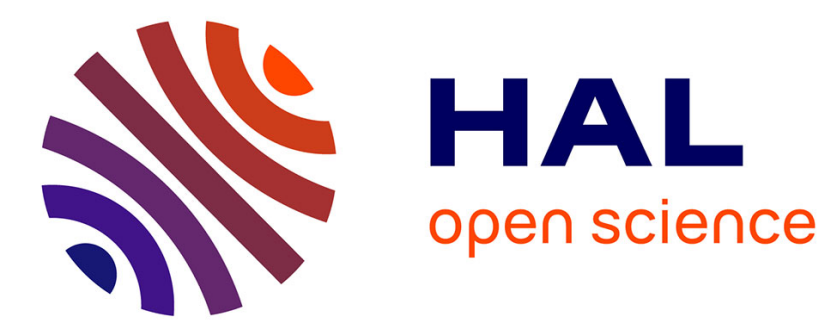

\title{
USNB: Enabling Universal Online Social Interactions
}

Rafael Angarita, Nikolaos Georgantas, Valérie Issarny

\section{To cite this version:}

Rafael Angarita, Nikolaos Georgantas, Valérie Issarny. USNB: Enabling Universal Online Social Interactions. 2017 IEEE 3rd International Conference on Collaboration and Internet Computing (CIC), Oct 2017, San Jose, United States. pp.309-318, 10.1109/CIC.2017.00048 . hal-01591757

\section{HAL Id: hal-01591757 \\ https://hal.inria.fr/hal-01591757}

Submitted on 21 Sep 2017

HAL is a multi-disciplinary open access archive for the deposit and dissemination of scientific research documents, whether they are published or not. The documents may come from teaching and research institutions in France or abroad, or from public or private research centers.
L'archive ouverte pluridisciplinaire HAL, est destinée au dépôt et à la diffusion de documents scientifiques de niveau recherche, publiés ou non, émanant des établissements d'enseignement et de recherche français ou étrangers, des laboratoires publics ou privés. 


\title{
USNB: Enabling Universal Online Social Interactions
}

\author{
Rafael Angarita*†§, Nikolaos Georgantas ${ }^{\dagger}$ and Valérie Issarny ${ }^{\dagger}$ \\ ${ }^{*}$ LISITE Laboratory. ISEP Paris, France \\ ${ }^{\dagger}$ MiMove Team. Inria Paris, France \\ rafael.angarita@isep.fr, firstname.lastname@inria.fr
}

\begin{abstract}
Online social network services (OSNSs) have become an integral part of our daily lives. At the same time, the aggressive market competition has led to the emergence of multiple competing siloed OSNSs that cannot interoperate. As a consequence, people face the burden of creating and managing multiple OSNS accounts and learning how to use them to stay connected. This paper is concerned with relieving users from such a burden by enabling universal online social interactions. The contributions of this paper span: (1) a model of the universal social network bus (USNB) for OSNS interoperability; (2) a prototype for universal online social interactions that builds upon the proposed model; and (3) a preliminary experimental evaluation involving 50 participants. Results show that people are positive about the solution as they are able to reach out a larger community of users independently of the OSNSs they use.
\end{abstract}

Index Terms-Online social networks, Online social interactions, Computer-mediated communication, Interoperability, Middleware, Service bus.

\section{Introduction}

Since humans -and other primates and animals- are social creatures, social networks have existed long before the Digital Age and still exist independently of it [1], [2], [3]. Social networks are social structures made up of a set of social actors, sets of dyadic ties, and other social interactions between actors. The emergence of the Internet has led to the creation of a plethora of software applications focused on social interactivity: from email in the 1960s and bulletin board systems in the 1970s, to Sixdegrees in 1997, and Facebook in 2004. Today, applications for social interactions are known as social networking services [4] or sites [5], social media [6], online social networks [7], or just social networks [8]. We adopt the term Online Social Network Service (OSNS) to refer to any digital platform allowing people to interact and build social relations.

A widely accepted definition of OSNSs is the one presented in [5], where authors define them as "webbased services that allow individuals to: (1) construct a

\footnotetext{
${ }^{\S}$ This work was done when the author was at Inria.
}

public or semi-public profile within a bounded system, (2) articulate a list of other users with whom they share a connection, and (3) view and traverse their list of connections and those made by others within the system". Other works such as [9] and [10] also emphasize the public or semi-public profile notion, which allows users to create a description of themselves, when defining OSNSs. These definitions exclude important non-profile-based services such as WhatsApp and email, for example. Indeed, researchers consider that messages exchanged by email users, and therefore users of any digital communication service, also form online social networks [11].

Since the emergence of OSNSs, the number of individuals using them has exploded. Some of the most popular OSNSs are Facebook, QQ, WeChat, QZone, Instagram, Tumblr, and Twitter. As of January 2017, and according to OSNSs statistics [12], there are more than 2,789 billion active OSNS users in the world. Further, this number grew by $21 \%$ in 2016, and it is expected to continue increasing. Still, the above statistics do not include widely used communication technologies such as email while it is estimated that the number of email users will increase to over 4.1 billion by the end of 2021 [13].

Another interesting fact is that, today, more than half of the Internet users (52\%) use two or more OSNSs [14]. One of the reasons behind these multiple memberships is the Fear of Missing Out [15], which is driven by the idea that others might be having rewarding experiences while one is absent and leads to the desire to stay continually connected. Other reasons relate to the specialization of some OSNSs (e.g., LinkedIn for professional use) that allow reaching different audiences [7], and/or the need to join other OSNSs to be able to connect with friends and family. A national survey in the U.S. shows that users are overwhelmed due to their multiple accounts [16], leading them to consider taking a break from one or more OSNSs. Not surprisingly, the same study found that most of the users wish there was a solution to the 
multiple membership problem.

OSNSs usually provide basic messaging functionalities such as private messages via instant messaging services or internal email, and public or semi-public message boards (also called user's wall or timeline) [7], [9]. These functionalities allowing OSNS users to interact can be classified as social interaction services [17], in contrast to other functionalities such as profile building. In general, we say that any system providing people the possibility of sending messages to other people or systems is a social interaction service, and thereby include communication technologies such as email, SMS and WhatsApp, just to mention some examples, in this classification.

The problem arises when a user of a particular social interaction service wants to interact with a user of a different one. In the case of decentralized open technologies such as email, both users must have an email account, but the email service provider does not matter. For closed proprietary platforms such as Facebook Messenger, both users are forced to have a Facebook Messenger account. Still, both cases require users to interact via the same social interaction service leading them to the undesired management of multiple accounts. Additionally, some social interaction services require users to be online at the same time to interact. Making different social interaction services interoperate also requires solving application data and middleware protocol mismatches. Moreover, the user interfaces of social interaction services only allow interacting with users of the same service. For instance, email users can only specify emails as the destination, while Facebook Messenger only shows other Facebook Messenger users in its contact list; therefore, a mechanism must be conceived to overcome this limitation. This is the issue we tackle in this paper, where we introduce an interoperability solution for social interaction services. More precisely, this paper features the following contributions:

- Background on interoperability of online social interactions. Section II introduces some OSNS research and describes existing practical efforts towards OSNS interoperability. This review concludes that no existing solution offers complete OSNS interoperability.

- A Universal Social Network Bus (USNB) model. Section III presents a model that captures user interactions across OSNSs via social interaction services. The model is based on the notion of social reachability, where the interaction capabilities of users are determined by the OSNSs they are registered in or logged in. The section further introduces the concept of persona to connect siloed OSNSs based on the service bus paradigm which was introduced by the distributed system community in the 2000s to overcome the arising heterogeneity among distributed services.

- Prototype implementation. Section IV showcases a working prototype of the proposed USNB, which provides interoperability between OSNSs for online social interactions. The prototype enables a variety of interactions between systems and people.

- Evaluation. Section V presents an experimental study involving 50 volunteers. Results show that participants are willing to use our solution for OSNS interoperability; provide feedback about what participants expect from such a solution; and highlight the feasibility of OSNS interoperability.

Finally, Section VI concludes with a summary of our contribution and future directions.

\section{Background}

There have been efforts to describe, characterize, and envision the future of OSNSs [4], [7], [17], [18]. These works are written narratively, so they do not propose models for OSNSs and their functionalities. On another note, the unprecedented availability of large amounts of data produced by OSNSs users has served as motivation for many researchers to understand OSNS user behavior. Other research is concerned with user identity spanning multiple OSNSs to understand the dangers of personal information leakage when having multiple OSNSs profiles [19], and to find a mapping among identities of individuals across OSNSs [20].

Regarding OSNSs interoperability, the existing solutions focus on enabling users to interact across OSNSs boundaries. One common solution to overcome heterogeneity is to introduce standards such as the ones proposed by the W3 Social Web Working Group ${ }^{1}$. However, the OSNS market is too competitive for providers to be willing to adopt a common standard [6]. On the contrary, many siloed OSNSs keep proliferating and gaining popularity. Thus, other solutions have emerged to overcome OSNS heterogeneity; they are oriented toward simplifying the management of multiple accounts:

- Account linkage. Account linkage is a well-known feature to share content across OSNSs. Users must possess accounts in all the OSNSs they are interested in and let the OSNS offering the account linkage feature access all the other accounts. It is usually limited to sharing content such as information found in news feeds. An example of account linkage is when users enable their Twitter

\footnotetext{
${ }^{1}$ https://www.w3.org/Social/WG
} 
accounts to access their Facebook accounts so that their Facebook wall posts are automatically published in their Twitter Timelines ${ }^{2}$. The advantage of this approach is that it lets users "stay" in their favorite OSNS and publish content in it while reaching users in other OSNSs.

- Aggregation service. Aggregation services collect content from multiple OSNSs to exhibit it as one unified presentation. As with account linkage, users have to give the aggregation service access to all the accounts they want to collect information from. In contrast with account linkage, aggregation services are third-party platforms that gather content, forcing users to leave their favorite OSNSs so as to benefit from content aggregation. friend2friend is an example of an aggregation service and brings together social content (photos, videos, stories) from Facebook, Twitter, YouTube, Instagram, etc.

- Universal instant messaging client. Universal instant messaging clients, similarly to aggregation services, provide a single interface to several messaging systems. This single interface also aggregates contact lists from the different social interaction services added to the client, giving the illusion of using one account to communicate with everybody no matter the social interaction service they are using. As with aggregation services, users not only have to possess accounts in all social interaction services they are interested in and give the client access to them, but they are also forced to "leave" them to enjoy the advantages of universal messaging. Pidgin is an example of a universal instant messaging client that supports many instant messaging protocols such as Bonjour, IRC, MySpaceIM, MSNP, OSCAR (AIM, ICQ, MobileMe, ...), XMPP/Jingle (Google Talk, LJ Talk, Gizmo5, ...), Yahoo! Messenger, etc.

- Distributed or federated social network. A distributed or federated social network [21] is an OSNS that is decentralized and distributed across distinct providers. It consists of a group of independently owned nodes, which coordinate to form the network. The created OSNS is not owned by anyone. Communication among nodes of the federation is conducted over social networking protocols. The software used for distributed social networking is generally portable so it is easily adopted on various website platforms. Distributed social network projects generally develop software, protocols, or both, which are released as

\footnotetext{
${ }^{2}$ https://www.facebook.com/help/1547895645497795?helpref= uf_permalink
}

open source most of the time. Open standards such as OAuth authorization, OpenID authentication, OStatus federation and the Extensible Messaging and Presence Protocol (XMPP) (aka Jabber) are some of the technologies for distributed social networking. Diaspora is an example of a userowned distributed social network.

Although the above solutions ease the management of multiple OSNSs accounts, they do not fully address interoperability across them. They either introduce yet another OSNS or enable coordinating specific functions between OSNSs. In our work, we are interested in enabling full interoperability across OSNSs. That is, we aim to let people interact beyond the boundaries of the OSNSs they use while relieving them from the burden of creating and managing multiple accounts and learning how to use new tools.

Toward that goal, we build upon the work in the distributed systems community [22]. More specifically, we adopt the service bus paradigm [23] which enables existing loosely coupled service components, possibly implemented using different technologies, to exchange messages transparently through an intermediary representation. Service components connect to the service bus, removing the need of solving interoperability problems between every pair of them.

In our previous work [24], we introduced a conceptualization of the human interaction paradigms in the virtual sphere and a service bus-like approach to decouple them and make them interoperate. This previous work introduces an initial idea for social communication interoperability, showcasing a one-way notification user case. In the present work, we build upon this idea to provide a model for OSNSs, deal with the challenges of siloed OSNSs and present an interoperability solution between users of different OSNSs.

To the best of our knowledge, despite the OSNS hype and the extensive research on interoperability, we are the first to introduce an overall approach, from design to implementation, for the interoperability of online social interactions. Thus, our work aims to fill the gap between interoperability and online social network research.

\section{USNB for OSNS interoperability}

\section{A. OSNSs and Social Interaction Services}

Social interaction services such as email, SMS, Facebook Messenger, Twitter, among others, are a fundamental part of OSNSs and computer-mediated communication, allowing individuals to interact in the virtual sphere.

Definition 1. A social interaction service, denoted $s$, is any functionality allowing users to send messages 
to other users or systems. A social interaction service $s$ can be used to both send and receive information, and it is modeled as a tuple $s=\langle$ name, $I, O, \tau\rangle$ where:

- name is a unique name representing $s$;

- $I$ is a set of inputs defining the information a user can receive using $s$ such that $I=$ $\left\{i_{1}, \ldots, i_{n}\right\}$, where each of these inputs takes the form $\langle$ name, type $\rangle$;

- $O$ is a set of outputs defining the information a user can send using $s$ such that $O=\left\{o_{1}, \ldots, o_{n}\right\}$, where each of these outputs takes the form 〈name, type ;

- $\tau$ indicates whether $s$ handles offline messaging ( $\tau=$ true) or users must be online at the same time to interact ( $\tau=$ false).

Typically, social interaction services are used to send and receive messages with the definition of $I$ and $O$ being the same. For example, the inputs and outputs of an email service can be defined as: $I=$ $O=\{\langle$ subject, MessageSubject $\rangle,\langle$ body, MessageBody \rangle$,\langle$ attachment, MessageAttachment $\rangle\}^{3}$.

The relationships among social entities in both the online and offline contexts can be represented with a graph [25], [26] where nodes represent individuals and edges represent relationships among them. The emergence of OSNSs has allowed the further study of these social network graphs. In particular, researchers have been interested in the two following graphs:

- Social graph [27], [8]: The representation of binary social links between users, e.g., friendship and family relationships.

- Activity graph [28], [29]: The representation of the interactions among users in an OSNS (as opposed to only capturing their social links).

The social and activity graphs are created dynamically according to the formation of social links and the interactions between users. However, these graphs do not reflect the central question of social communication interoperability: "whom can an OSNS user interact with?". To illustrate this question, we introduce the notion of social reachability graph.

Definition 2. Let $U$ be the universe of OSNS users. $U$ can be partitioned into connected multigraphs, called social reachability graphs and each denoted $R=$ $(V, E)$, where $V \subseteq U$, with the following properties:

- Every edge $<u_{i}, u_{j}>\in E$, with $u_{i}, u_{j} \in V$, has an associated social interaction service, $s \in S$, such that both $u_{i}$ and $u_{j}$ are users of $s$.

- For any given pair of users $u_{0}, u_{k} \in V$, there exists at least one bidirectional path $\mathcal{P}$ such that:

\footnotetext{
${ }^{3}$ Types can be concepts associated to an ontology.
}

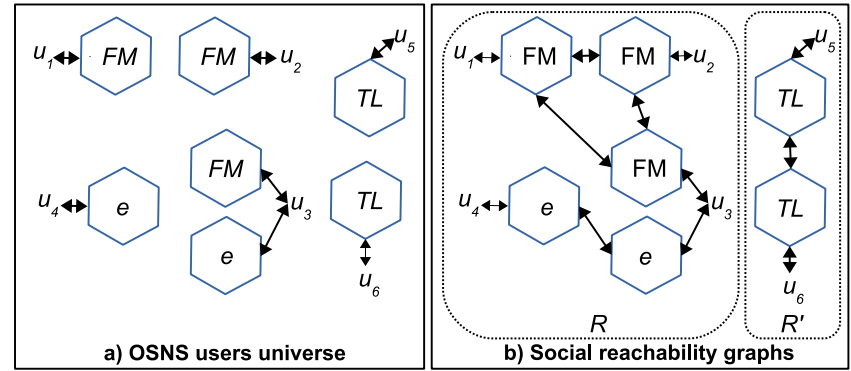

Fig. 1. Social reachability graph

$$
\mathcal{P}\left(u_{0}, u_{k}\right)=u_{0} \underset{s_{0,1}}{\stackrel{s_{1,0}}{\leftrightarrows}} u_{1} \underset{s_{1,2}}{\stackrel{s_{2,1}}{\leftrightarrows}} u_{2} \underset{s_{\ldots, 2}}{\stackrel{s_{2}, \ldots}{\leftrightarrows}} \ldots u_{k-1} \underset{s_{k, k-1}}{\leftrightarrows} u_{k}
$$

where

$s_{0,1}, s_{1,0}, s_{1,2}, s_{2,1}, s_{\ldots, 2}, s_{2, \ldots}, \ldots, s_{k, k-1}, s_{k-1, k}$

are social interaction services associated to the corresponding edges.

Users in the same social reachability graph can interact. Figure 1 (a) shows a universe of OSNS users, $U=\left\{u_{1}, u_{2}, u_{3}, u_{4}, u_{5}, u_{6}\right\}$, involving Facebook Messenger $(F M)$, email $(e)$, and Twitter Timeline $(T L)$. Figure 1 (b) shows how $U$ is partitioned into two social reachability graphs, $R$ and $R^{\prime}$, by Def. 2. Users in $R$ cannot reach users in $R^{\prime}$ and vice-versa.

In addition to the social reachability graph barrier, another phenomenon hindering interaction is the log in and out behavior of users, which defines the user presence in a given social interaction service.

Definition 3. The user presence of a user $u$ in a social interaction service $s$ at time $t \in T_{s}$, where $T_{s}$ is an interval of time when $s$ is available, is modeled as an alternating renewal process, as follows:

$$
Z_{u, s}(t)= \begin{cases}\text { true, } & \text { if user } u \text { is logged in } s \text { at time } t \\ \text { false, } & \text { otherwise }\end{cases}
$$

Definition 4. The interaction conditions for two OSNS users, $u$ and $v$, are the following:

- $u$ and $v$ are in the same social reachability graph:

$$
u, v \in V(R)
$$

- There exist a path $\mathcal{P}(u, v)$, between $u$ and $v$, such that all pairs of adjacent users $u_{i}$ and $v_{j}$ in $\mathcal{P}(u, v)$ can interact via $s_{u_{i}, v_{j}}$ : 


$$
\begin{aligned}
& \exists \mathcal{P}(u, v) \in R: \\
& \forall<u_{i}, v_{j}>\in E(\mathcal{P}(u, v)), \\
& \exists t_{k} \in T_{s_{u_{i}, v_{j}}}:\left(Z_{u_{i}, s_{u_{i}, v_{j}}}\left(t_{k}\right) \wedge Z_{v_{j}, s_{u_{i}, v_{j}}}\left(t_{k}\right)\right) \\
& \vee \\
& \tau_{s_{u_{i}, v_{j}}}
\end{aligned}
$$

\section{B. Universal Social Network Bus}

We present the universal social network bus (USNB) as the fundamental component to enable universal online social interactions.

Definition 5. The universal social network bus (USNB), denoted $\mathcal{B}$, is an entity allowing to integrate siloed OSNSs over a service bus-like paradigm by featuring a reference abstract social interaction service, $s_{\mathcal{B}}$, and enacting a social reachability graph, $R_{\mathcal{B}}$.

The main components of USNB are called USNB personae, or simply personae. Personae act as bridges between any plugged OSNS and USNB.

Definition 6. A USNB persona, denoted $p$, is an entity connecting $\mathcal{B}$ and a concrete OSNS $n$ such that:

- $p$ is in the social reachability graphs $R_{\mathcal{B}}$ (of $\mathcal{B}$ ) and $R_{n}$ (of $n$ ):

$$
p \in V\left(R_{\mathcal{B}}\right) \wedge p \in V\left(R_{n}\right)
$$

- $p$ is present in $s_{\mathcal{B}}$ :

$$
\forall t \in T_{s_{\mathcal{B}}}: Z_{p, s_{\mathcal{B}}}(t)
$$

- $p$ is present in a social interaction service, $s$, of $R_{n}$ :

$$
\exists s \in E\left(R_{n}\right), \forall t \in T_{s}: Z_{p, s}(t)
$$

The linking of personae allows users from isolated social reachability graphs to interact independently of their technological choices, as depicted in Figure 2. Zooming in on $p^{\prime \prime}$, the figure illustrates the representation of users in other OSNSs within $p^{\prime \prime}$. Any two personae $p, p^{\prime} \in V\left(R_{\mathcal{B}}\right)$ can interact via $s_{\mathcal{B}}$; therefore, for any two users $u$ and $v$, there exists a path such that:

$$
\mathcal{P}(u, v)=u \underset{s_{i}}{\stackrel{s_{i}}{\leftrightarrows}} p \underset{s_{\mathcal{B}}}{\stackrel{s_{\mathcal{B}}}{\leftrightarrows}} p^{\prime} \underset{s_{j}}{\stackrel{s_{j}}{\leftrightarrows}} v
$$

What happens between $p$ and $p^{\prime}$ in eq. (1) is transparent for $u$ and $v$. From $u^{\prime}$ s perspective, interactions happen only via $s_{i}$; from the one of $v$, only via $s_{j}$.

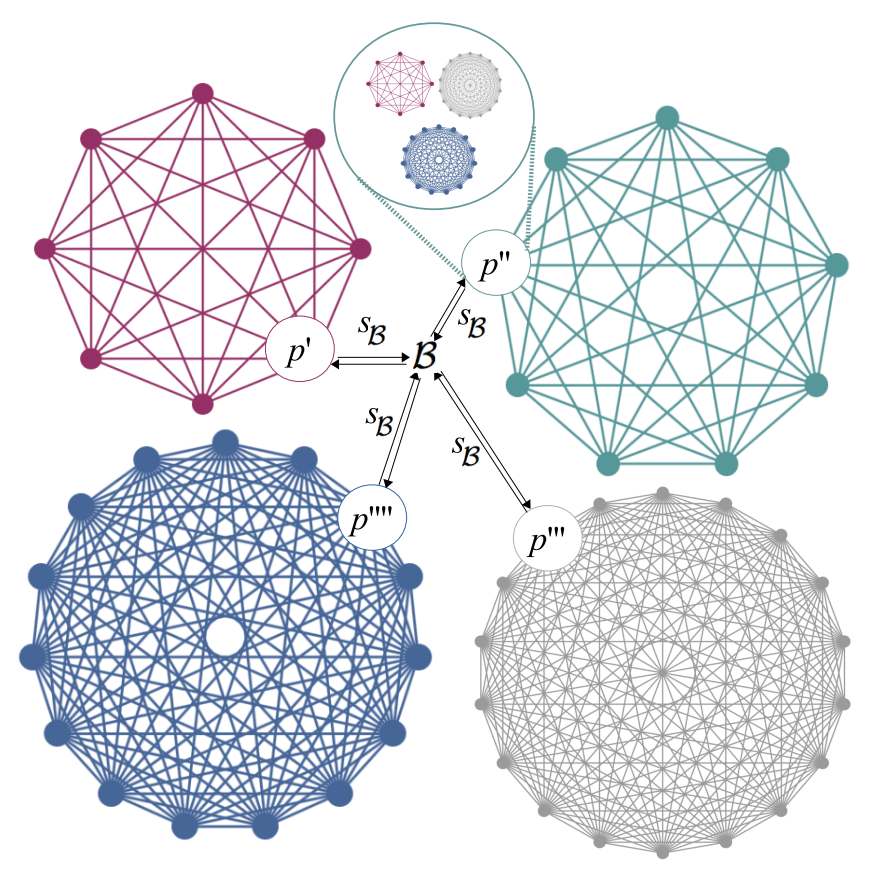

Fig. 2. Connecting siloed OSNS users via personae

\section{Illustrative use case}

Consider five individuals using different social interaction services: (1), Carmen uses Facebook Messenger; (2), John prefers email; (3), Clara communicates via Twitter Direct Messages; (4), Silvia, although she also uses Twitter, she prefers to maintain open and short conversations with Twitter Timeline; and (5), Jane prefers Slack Private Messages. John wants to send a message to all of them without leaving his favorite email client. Then, how does John do that? Suppose the existence of the following personae:

- $p_{\digamma}$ is a Facebook persona using Facebook Messenger $F M$ such that:

$$
p_{\digamma} \in V\left(R_{F M}\right) \wedge p_{\digamma} \in V\left(R_{\mathcal{B}}\right)
$$

- $p_{\top}$ is a Twitter persona using Twitter Direct Messages $D M$ and Twitter Timeline $T L$ such that:

$$
p_{\top} \in V\left(R_{D M}\right) \wedge p_{\top} \in V\left(R_{T L}\right) \wedge p_{\top} \in V\left(R_{\mathcal{B}}\right)
$$

- $p_{\sigma}$ is a Slack persona using Slack Private Messages $P M$ and Slack Channels $C H$ such that:

$$
p_{\sigma} \in V\left(R_{P M}\right) \wedge p_{\sigma} \in V\left(R_{C H}\right) \wedge p_{\sigma} \in V\left(R_{\mathcal{B}}\right)
$$

- $p_{\supset}$ is a Gmail persona using email $e$ such that:

$$
p_{\supset} \in V\left(R_{e}\right) \wedge p_{\supset} \in V\left(R_{\mathcal{B}}\right)
$$


Since all users are in $V\left(R_{\mathcal{B}}\right)$, there exists a path $\mathcal{P}$ between all of them; hence, John can interact with other users via $p_{\supset}$ using his favorite email client:

$$
\begin{aligned}
& \mathcal{P}(\text { John, Carmen })=\text { John } \underset{e}{\stackrel{e}{\leftrightarrows}} p_{\supset} \underset{s_{\mathcal{B}}}{\stackrel{s_{\mathcal{B}}}{\leftrightarrows}} p_{\digamma} \underset{F M}{\stackrel{F M}{\leftrightarrows} \text { Carmen }} \\
& \mathcal{P}(\text { John, Clara })=\text { John } \underset{e}{\stackrel{e}{\leftrightarrows}} p_{\supset} \underset{\mathcal{B}_{\mathcal{B}}}{\stackrel{s_{\mathcal{B}}}{\leftrightarrows}} p_{\top} \underset{D M}{\stackrel{D M}{\leftrightarrows} \text { Clara }} \\
& \mathcal{P}(\text { John, Silvia })=\text { John } \underset{e}{\stackrel{e}{\leftrightarrows}} p_{\supset} \underset{s_{\mathcal{B}}}{\stackrel{s_{\mathcal{B}}}{\leftrightarrows}} p_{\top} \underset{T L}{\stackrel{T L}{\leftrightarrows}} \text { Silvia } \\
& \mathcal{P}(\text { John, Jane })=\text { John } \underset{e}{\stackrel{e}{\leftrightarrows}} p_{\supset} \underset{s_{\mathcal{B}}}{\stackrel{s_{\mathcal{B}}}{\leftrightarrows}} p_{\sigma} \underset{P M}{\stackrel{P M}{\leftrightarrows}} \text { Jane }
\end{aligned}
$$

Similarly, the rest of the users can also interact with the personae corresponding to their social interaction services to reach all other users.

\section{USNB prototype implementation}

\section{A. Architecture}

The architecture of USNB is shown in Figure 3. Its core service components are the following:

- Entity Manager is a registry for users, personae, and user OSNS identities and preferences. Each user has one or more identities associated to existing personae. For example, a user $u$ can have an email address and a Facebook Messenger id. $u$ can also choose one of his two identities as his preferred one, so he will only receive messages using the social interaction service corresponding to that identity.

- Subscription Manager maintains a list of events, subscriptions to those events, and has an endpoint to send messages related to those events. It can be used to implement group communication or followlike functionalities as we presented in [24].

- Message-oriented middleware supports asynchronous communication between the distributed service components of USNB.

Both Entity Manager and Subscription Manager offer REST APIs to interact with USNB.

Personae are in charge of translating messages and coordinating behavior between social interaction services and USNB. Figure 3 shows the following personae: Facebook Messenger $\left(p_{\digamma}\right)$, Twitter Direct Messages and Twitter Timeline $\left(p_{\top}\right)$, Gmail $\left(p_{\supset}\right)$, Slack private messages and channel-based messages $\left(p_{\sigma}\right)$, and the AppCivist platform for participatory democracy and budgeting [30] $\left(p_{a p p}\right)$. Additional personae can be plugged into USNB to enable interactions with other systems or OSNSs, and they can be implemented manually or dynamically synthesized [31].

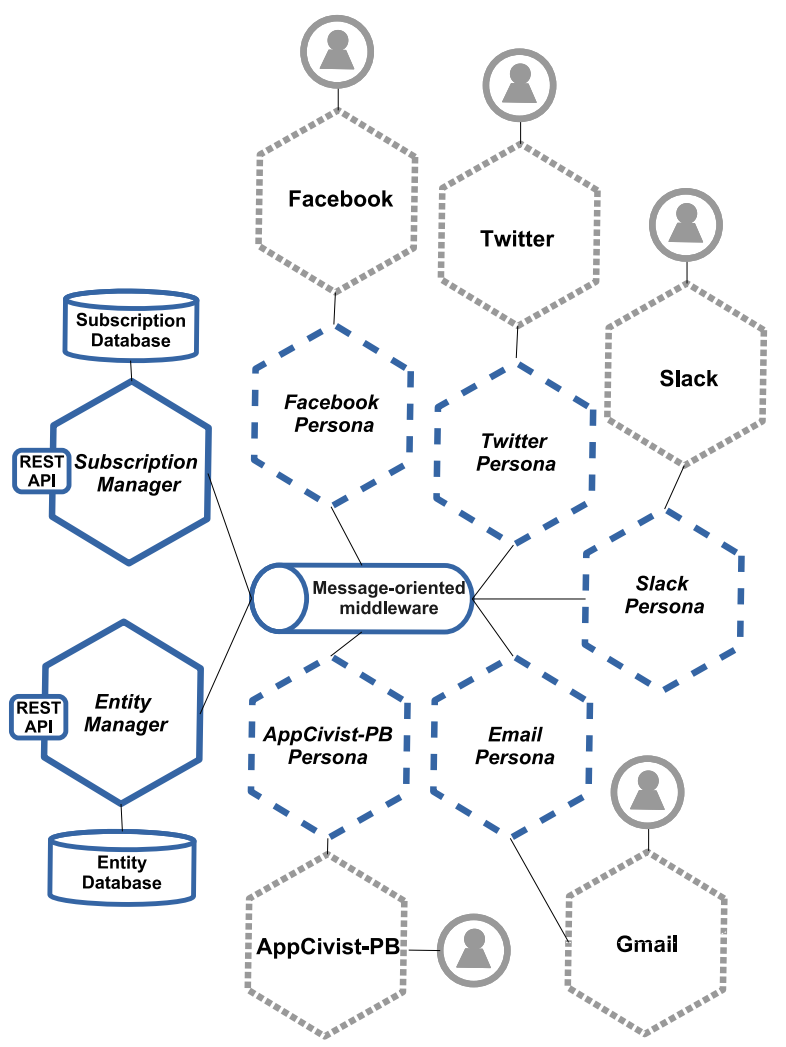

Fig. 3. System prototype

\section{B. USNB implementation}

Our current prototype implements all the services, including personae, in Node.js, while the databases are implemented using MongoDB. RabbitMQ is used as the message-oriented middleware implementation for inter-service communication. All services have an AMQP endpoint and a client to receive and send messages in the format showed in Listing 1. to and from specify receivers and senders, respectively, which are defined by their unique names and can have a screen name. message can be used to specify message parts such as text and subject. This message format allows USNB to be compatible with most social interaction services. This message is sent and received in the AMQP message body and is written as a set of data objects consisting of attribute-value pairs using the JSON format.

Listing 1. Example USNB messaqe

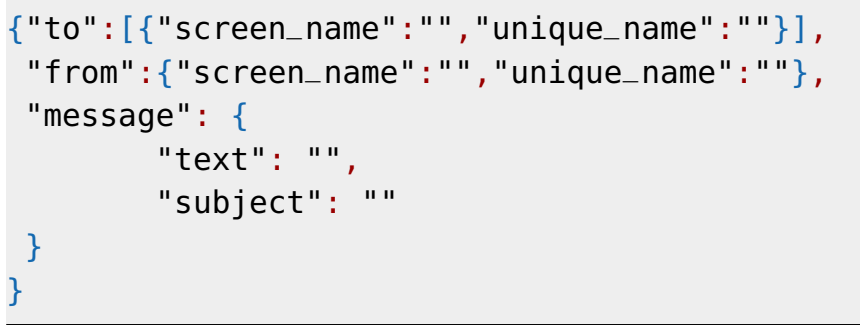




\section{Personae instances}

Users may have to follow different procedures to interact with the persona of the social interaction service they use. Facebook Messenger users who are not friends with $p_{\digamma}$ will receive a conversation invitation when receiving a message from it for the first time. They must accept the invitation to let $p_{\digamma}$ communicate with them. They can also send a message to $p_{\digamma}$ to allow communication. Users of Twitter Direct Messages have two options to let $p_{\top}$ communicate with them: (i), follow it; (ii), go to settings, privacy and safety, Direct Messages, and check the "Receive Direct Messages from anyone" box. Users of Twitter Timeline do not have to do anything in particular. Since Slack is a team-based collaboration tool, users have to add $p_{\sigma}$ to their teams using the Slack App Directory to communicate with it via private messages or channel-based messages. Thanks to the distributed nature of email, and the fact that Gmail is a well-known service, email users are able to communicate with $p_{\partial}$ right away.

To deal with the inadaptability of the text-based user interfaces of the various social interaction services, all personae of the current prototype enable users to interact with USNB and USNB entities via commands beginning with a number sign (\# $)^{4}$. Currently, the available functionalities are the following:

- Getting general information about USNB such as what it is for, its current version, license and contacts:

\#about

- Getting help concerning the USNB usage and interaction with the corresponding persona: \#help

- Creating a USNB account or linking the identity of the current social interaction service to an existing USNB account:

\#login

- Setting the identity of the current social interaction service as the preferred one:

\#prefer

- Getting the list of USNB entities. Entities can be people, system or personae:

$\# 1 \mathrm{~s}$

- Getting information about a specific entity: \#more entity

\footnotetext{
${ }^{4}$ Commands are increasingly used to enable users to interact with OSNSs; for example, see https://api.slack.com/slash-commands.
}

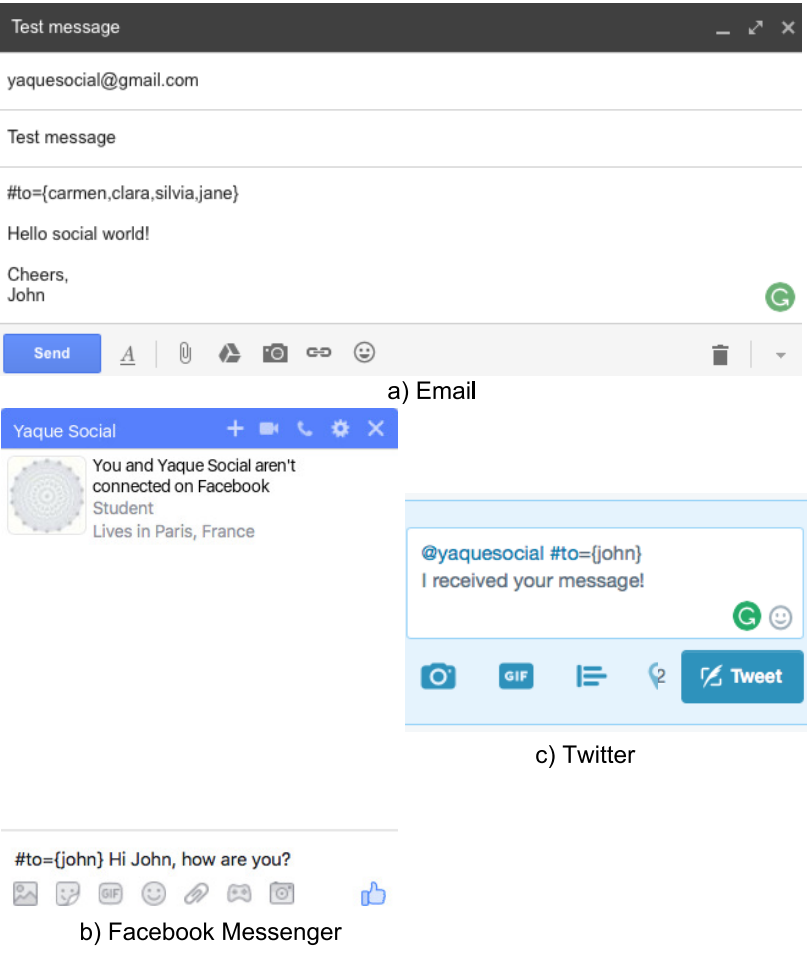

Fig. 4. Sending a message using different social interaction services

- Sending a message to a list of one or more entities:

$\#$ to $=\{$ list $\}$

These commands can appear in any part of a message; for example, an email user may write the \#to $=\{$ list $\}$ command in the message subject or wherever in the message body. Note that other implementations of personae can use this commands as the underlying functions for implementing them in other languages, variations of the commands, or in natural language.

Figure 4 illustrates sending a message with three different social interaction services. The instance of USNB showed in this example is called Yaque. Using email (Figure 4 (a)), users have to specify the email address of $p_{\supset}$. In Facebook Messenger (Figure 4 (b)), and in all instant messaging interfaces such as Slack Private Messages and Twitter Direct Messages, users only have to open a conversation window with the corresponding persona. For Twitter Timeline (Figure 4 (c)), users have to mention $p_{\top}$ in a tweet. Figure 5 illustrates the message reception with different social interaction services.

Note that personae do not ask users their credentials nor any kind of authorization regarding their OSNSs accounts. 


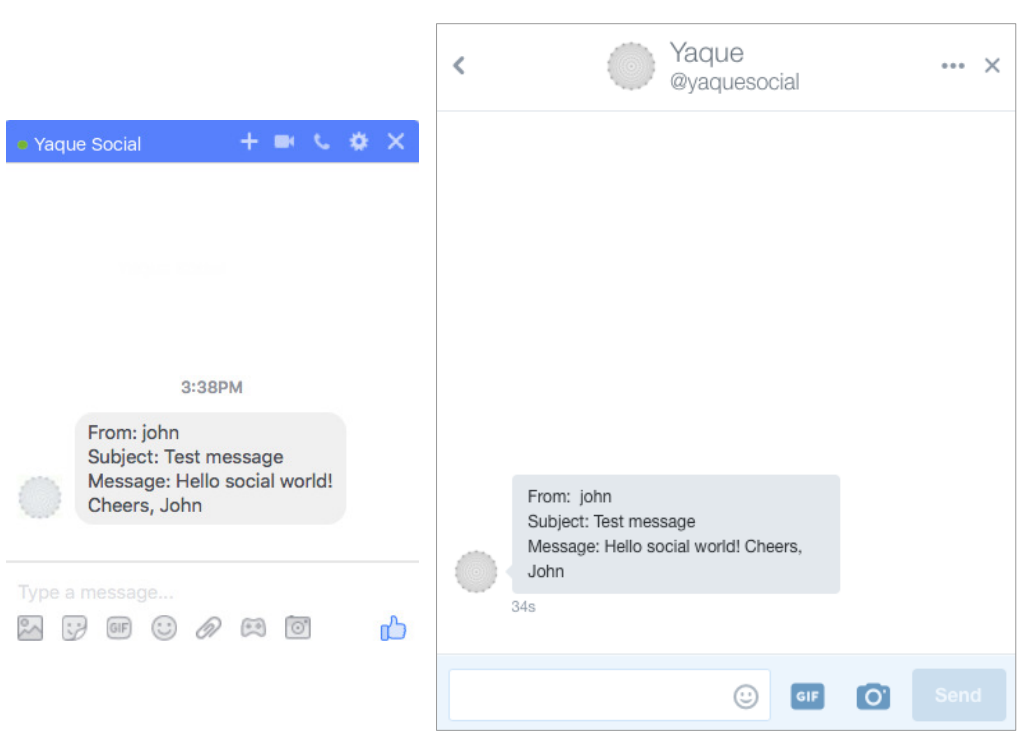

a) Facebook Messenger

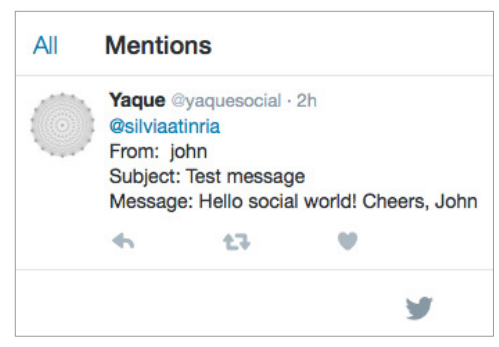

c) Twitter Timeline

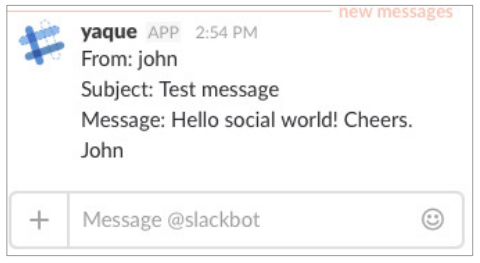

d) Slack

Fig. 5. Message reception by heterogeneous social interaction services

\section{Evaluation \& assessment}

\section{A. Rationale}

The feasibility of universal online social interactions was demonstrated by the prototype of Section IV. The process of transforming application data, adapting middleware protocols and coordinating functions of social interaction services and USNB is transparent to users. A key issue is that the user interfaces of social interaction services cannot be adapted to this interoperability context. This is the reason why users have to tell personae what they want to do using the existing and immutable user interfaces. In the current prototype, users do this via commands. This solution impacts the user experience for online social interactions; consequently, the main objectives of this evaluation are the following: (i), find out if users are willing to interact with personae for the sake of OSNS interoperability; and (ii), understand what users expect from this interaction and how they would like it to be.

\section{B. Participants}

A total of 50 participants experimented with USNB. Participants came from diverse backgrounds and 15 countries, and their age range was 18-70 years. We asked them to choose one social interaction service out of the five available in the implementation and use their own personal account for the experiments when they had one. If requested, we provided a test account for the chosen social interaction service. Participants were briefly taught the idea behind USNB and how to use the current prototype. They were encouraged to think about how they would like to interact with personae based on the presented functionality.

\section{Procedure}

Participants were given the task of communicating with a user who does not use their chosen social interaction service by using the implemented commands. To achieve that, participants were provided with instructions and help on how to use these commands, and how to find the persona corresponding to their chosen social interaction service. At the end of the experience, participants completed a survey consisting of general background information and the USE questionnaire (Usefulness, Satisfaction, and Ease of use) [32] to obtain feedback regarding the interactions with personae. Participants were explicitly asked what are the positive and negative points of interacting with personae and how they would prefer this interaction to be. Participants sent their answers directly to USNB via $\rho_{\curvearrowright}$ (i.e., by email). USNB also kept a log to get insights about its usage; e.g, which commands users tried and how successful they were using $\mathrm{USNB}^{5}$.

\section{Results}

We found out that the participants tried the \#to command an average of 2.46 times before succeeding. In the best case, $30.76 \%$ of participants succeed at the first try; in the worst case, $15.38 \%$ of participants succeed after 6 tries. Also, 84.61\% of participants explored other commands such as \#ls, \#more, \#help,

\footnotetext{
${ }^{5}$ Completed surveys (without family names), logs, and additional information can be found at https://tinyurl.com/lelxz2j
} 


\begin{tabular}{llll}
\hline & agree (\%) & neutral (\%) & disagree (\%) \\
\hline usefulness & 91.66 & 5 & 2.5 \\
ease of use & 67.87 & 23.63 & 7.27 \\
satisfaction & 86.66 & 4.66 & 6.00 \\
\hline
\end{tabular}

Simplified view of the survey results where agree corresponds to rates from 5 to 7 , neutral to rate 4, and disagree to rates from 1 to 3 , and percentages are the averages of questions in each category

and \#about. $15.38 \%$ of participants wanted to add the Facebook persona as a friend. Finally, $38.46 \%$ of participants tried to interact with the personae using natural language even though they were informed that they could only use commands to interact with personae. As a conclusion from the logs, all users were successful relatively easily despite the brief exposure to USNB and the low-level mechanism to interact with personae. This is an encouraging outcome since we presented them with basic command-like functionalities; these functionalities can be extended and used to build services on top of them to offer a richer user experience.

Regarding the USE questionnaire, participants rated our solution high in usefulness and satisfaction, as seen in Table I. We did not include the ease of learning section of the questionnaire since users are accessing directly the functionality of the USNB prototype only as a demonstration.

There were interesting suggestions about the organization of individual conversations with multiple users using Facebook Messenger, Twitter Direct Messages, and Slack. This problem does not exist with email since message threads are visually grouped by subject, where the subject can be the name of the user sending the message, for example. It is neither a problem with Twitter Time Line since the Twitter interface shows messages separately and users do not expect to use this Twitter functionality to have twoway conversations as in instant messaging applications. Slack offers interesting functionalities such as Message Threads to organize conversations, which can be exploited by the corresponding persona. A positive remark regarding trust and privacy is that users do not have to give access to their accounts, contrary to existing solutions for account linkage, aggregation services and universal chat clients. As a conclusion from the USE questionnaire, participants thought that it will be great and useful to adopt our solution to avoid creating and managing multiple OSNSs accounts and that the user experience will greatly improve by giving natural language capabilities to the personae.

\section{E. Limitations}

One limitation concerns the rate limiting imposed by OSNSs providers such as Twitter ${ }^{6}$, Facebook $^{7}$, and Gmail $^{8}$. Personae can also be blocked by an OSNS provider if they are perceived as performing spamlike activity ${ }^{9}$. Another risk is that OSNSs APIs can change; in this case, an adaptation or regeneration of the concerned persona is required. A specific issue is that Twitter Timeline does not allow sending the exact same tweet more than once. This is usually not a problem when users interact between them; however, it is not possible for a user to send, for example, the tweet @yaquesocial \#ls two times consecutively. As a workaround, users, as well as USNB, can change the message by writing some extra text; for example, ayaquesocial \#ls this means nothing!.

\section{F. Assessment}

We have demonstrated with our prototype that OSNS interoperability is not only possible, but also can soon become widely available. Our prototype highlighted the technical challenges of OSNS interoperability, which depend on restrictions imposed by OSNS providers and on the user interface constraints of different social interaction services. The experimental evaluation showed that not only users are willing to use our solution, but they also thought it was fun. Users also gave feedback about how they would like to interact with our prototype, which can serve as a foundation for building a production-level solution for OSNS interoperability.

\section{Conclusions and Future Work}

We presented a novel model of OSNSs which allows to understand and illustrate the OSNS interoperability problem, and devise a solution from design to prototype implementation. Our approach proved that OSNS interoperability is possible and highlighted its challenges. A group of participants tested our prototype. Their feedback was positive, confirming its usefulness. Participants also reported suggestions to improve user experience and ideas of additional functionalities. Enabling social reachability among users of siloed OSNS diminishes -or removes- the need of creating multiple OSNSs accounts. As a practical consequence, our contribution has the potential of influencing the OSNS landscape.

\footnotetext{
${ }^{6} \mathrm{https}: / /$ dev.twitter.com/rest/public/rate-limiting

${ }^{7}$ https://developers.facebook.com/docs/graph-api/advanced/ratelimiting

${ }^{8} \mathrm{https}: / /$ developers.google.com/gmail/api/v1/reference/quota

${ }^{9}$ https://twittercommunity.com/t/error-code-226-this-request-lookslike-it-might-be-automated/12872
} 
Our work opens many thrilling perspectives such as studying privacy concerns and end-to-end perception of interactions across OSNSs, and the automatic synthesis of personae. Concerning the implementation, we plan to continue the developing of personae to enhance user experience with functionalities such as user searching, conversation management, interactions with personae in natural language, and crossOSNS access control.

\section{Acknowledgments}

This work is partially supported by the Inria Project Lab CityLab (citylab.inria.fr) and the Inria@SiliconValley program (project.inria.fr/siliconvalley). The authors also acknowledge the support of the CivicBudget activity of EIT Digital (www.eitdigital.eu).

\section{References}

[1] R. A. Hill and R. I. M. Dunbar, "Social network size in humans," Human Nature, vol. 14, no. 1, pp. 53-72, 2003.

[2] H. Kudo and R. I. Dunbar, "Neocortex size and social network size in primates," Animal Behaviour, vol. 62, no. 4, pp. 711 722, 2001.

[3] D. Lusseau and M. E. Newman, "Identifying the role that animals play in their social networks," Proceedings of the Royal Society of London B: Biological Sciences, vol. 271, no. Suppl 6, pp. S477-S481, 2004.

[4] J. Breslin and S. Decker, "The Future of Social Networks on the Internet: The Need for Semantics," IEEE Internet Computing, vol. 11 , no. 6, pp. 86-90, Nov 2007

[5] N. B. Ellison et al., "Social network sites: Definition, history, and scholarship," Journal of Computer-Mediated Communication, vol. 13, no. 1, pp. 210-230, 2007.

[6] D. Hinchcliffe, "Where is interoperability for social media?" Enterprise Web 2.0, 2014. [Online]. Available: http://www.zdnet. com/where-is-interoperability-for-social-media-7000026894/

[7] J. Heidemann, M. Klier, and F. Probst, "Online social networks: A survey of a global phenomenon," Computer Networks, vol. 56, no. 18, pp. 3866-3878, 2012.

[8] C. Wilson, B. Boe, A. Sala, K. P. Puttaswamy, and B. Y. Zhao, "User Interactions in Social Networks and Their Implications," in Proceedings of the 4th ACM European Conference on Computer Systems, ser. EuroSys '09. New York, NY, USA: ACM, 2009, pp. 205-218.

[9] F. Schneider, A. Feldmann, B. Krishnamurthy, and W. Willinger, "Understanding online social network usage from a network perspective," in Proceedings of the 9th ACM SIGCOMM conference on Internet measurement conference. ACM, 2009, pp. $35-48$.

[10] A. Armstrong and J. Hagel, III, "Creating Value in the Network Economy," D. Tapscott, Ed. Boston, MA, USA: Harvard Business School Press, 1999, ch. The Real Value of On-line Communities, pp. 173-185.

[11] A. Mislove, M. Marcon, K. P. Gummadi, P. Druschel, and B. Bhattacharjee, "Measurement and Analysis of Online Social Networks," in Proceedings of the 7th ACM SIGCOMM Conference on Internet Measurement, ser. IMC '07. New York, NY, USA: ACM, 2007, pp. 29-42.

[12] D. Chaffey, "Global social media research summary 2017," http://www.smartinsights.com/social-media-marketing/ social-media-strategy/new-global-social-media-research/, 2017.

[13] T. R. Group, “Email Statistics Report, 2017-2021," http://www. radicati.com/?p=14588, 2017.
[14] M. Duggan, N. B. Ellison, C. Lampe, A. Lenhart, and M. Madden, "Frequency of social media use," http://www.pewinternet.org/ 2015/01/09/frequency-of-social-media-use-2/, 2015.

[15] A. K. Przybylski, K. Murayama, C. R. DeHaan, and V. Gladwell, "Motivational, emotional, and behavioral correlates of fear of missing out," Computers in Human Behavior, vol. 29, no. 4, pp. 1841 - 1848, 2013.

[16] MyLife.com, "Connecting and Communicating Online: State of Social Media," http://photos.prnewswire.com/prnfull/ 20130709/DE44018-INFO, 2013.

[17] H. Quan, J. Wu, and Y. Shi, "Online Social Networks and Social Network Services," in Pervasive Communications Handbook. CRC Press, 2011, pp. 1-22.

[18] A. Pathak, G. Rosca, V. Issarny, M. Decat, and B. Lagaisse, Privacy and Access Control in Federated Social Networks. Cham: Springer International Publishing, 2014, pp. 160-179.

[19] D. Irani, S. Webb, K. Li, and C. Pu, "Modeling unintended personal-information leakage from multiple online social networks," IEEE Internet Computing, vol. 15, no. 3, pp. 13-19, 2011.

[20] R. Zafarani and H. Liu, "Connecting Users Across Social Media Sites: A Behavioral-modeling Approach," in Proceedings of the 19th ACM SIGKDD International Conference on Knowledge Discovery and Data Mining, ser. KDD '13. New York, NY, USA: ACM, 2013, pp. 41-49.

[21] R. Esguerra, "An Introduction to the Federated Social Network," https://www.eff.org/deeplinks/2011/03/ introduction-distributed-social-network, 2011.

[22] V. Issarny, M. Caporuscio, and N. Georgantas, "A Perspective on the Future of Middleware-based Software Engineering," in 2007 Future of Software Engineering, ser. FOSE '07. Washington, DC, USA: IEEE Computer Society, 2007, pp. 244-258.

[23] D. Chappell, Enterprise service bus. O’Reilly Media, Inc., 2004.

[24] R. Angarita, N. Georgantas, C. Parra, J. Holston, and V. Issarny, "Leveraging the Service Bus Paradigm for Computer-mediated Social Communication Interoperability," in Proceedings of the 39th International Conference on Software Engineering: Software Engineering in Society Track, ser. ICSE-SEIS '17, 2017, pp. 67-75.

[25] A.-L. Barabási, "Scale-Free Networks: A Decade and Beyond," Science, vol. 325, no. 5939, pp. 412-413, 2009

[26] S. Wasserman and K. Faust, Social network analysis: Methods and applications. Cambridge university press, 1994, vol. 8.

[27] F. Benevenuto, T. Rodrigues, M. Cha, and V. Almeida, "Characterizing User Behavior in Online Social Networks," in Proceedings of the 9th ACM SIGCOMM Conference on Internet Measurement, ser. IMC '09. New York, NY, USA: ACM, 2009, pp. 49-62.

[28] J. Heidemann, M. Klier, and F. Probst, "Identifying Key Users in Online Social Networks: A PageRank Based Approach," in 31st International Conference on Information Systems (ICIS), 2010.

[29] A. Nazir, S. Raza, and C.-N. Chuah, "Unveiling Facebook: A Measurement Study of Social Network Based Applications," in Proceedings of the 8th ACM SIGCOMM Conference on Internet Measurement, ser. IMC '08. New York, NY, USA: ACM, 2008, pp. 43-56.

[30] A. Pathak, V. Issarny, and J. Holston, "AppCivist - A Serviceoriented Software Platform for Socially Sustainable Activism," in International Conference on Software Engineering (ICSE), Software Engineering in Society (SEIS) Track, Florence, Italy, May 2015.

[31] R. Calinescu et al., Synthesis and Verification of Self-aware Computing Systems. Cham: Springer International Publishing, 2017, pp. 337-373.

[32] A. M. Lund, "Measuring Usability with the USE Questionnaire," Usability and User Experience, vol. 8, no. 2, pp. 3-6, 2001. 